\title{
Quasi-Static Problem of Thermoelasticity for Thermosensitive Infinite Circular Cylinder of Complex Heat Exchange
}

\author{
Halyna Harmatij ${ }^{1}$, Marta Król ${ }^{2}$, Vasyl Popovycz ${ }^{1}$ \\ ${ }^{1}$ Pidstryhach Institute for Applied Problems of Mechanics and Mathematics National Academy of Sciences of Ukraine, \\ Lviv, Ukraine \\ ${ }^{2}$ Faculty of Mathematics and Applied Physics, Rzeszow University of Technology, Rzeszow, Poland \\ Email: dept19@iapmm.lviv.ua,krolmb@prz.edu.pl
}

Received January 20, 2013; revised March 10, 2013; accepted April 13, 2013

Copyright (C) 2013 Halyna Harmatij et al. This is an open access article distributed under the Creative Commons Attribution License, which permits unrestricted use, distribution, and reproduction in any medium, provided the original work is properly cited.

\begin{abstract}
Nonlinear nonstationary heat conduction problem for infinite circular cylinder under a complex heat transfer taking into account the temperature dependence of thermophysical characteristics of materials is solved numerically by the method of lines. Directing it to the Cauchy's problem for systems of ordinary differential equations studied feature which takes place on the cylinder axis. Taken into account the dependence on the temperature coefficient of heat transfer that the different interpretation of its physical content makes it possible to consider both convective and convective-ray or heat ray. Using the perturbation method, the corresponding thermoelasticity problem taking into account the temperature dependence of mechanical properties of the material is construed. The influence of the temperature dependence of the material on the distribution of temperature field and thermoelastic state of infinite circular cylinder made of titanium alloy Ti-6Al-4V by radiant heat transfer through the outer surface has been analyzed.
\end{abstract}

Keywords: Thermoelastic State; Heat Transfer; Boundary Value Problem

\section{Introduction}

It is of interest to consider the condition of convective heat transfer coefficient depending on the temperature of heat transfer

$$
\left[\lambda_{t}(t) \frac{\partial t}{\partial n}+\alpha(t)\left(t-t_{c}\right)\right]_{S}=0
$$

Here $\lambda_{t}(t)$-coefficient of thermal conductivity, which depends on the temperature; $t_{c}$-temperature environment, which washes the body surface $S ; n$-external normal to the surface $S$. That kind of heat transfer conditions also considers heating or cooling the body by radiation, or heat when carried out simultaneously by convection and radiation. Thus the physical content of Equation (1) is quite different. For radiation heat transfer coefficient $\alpha_{t}$ is called the coefficient of radiation heat transfer, it has the same dimension as the coefficient of convective heat transfer is:

$$
\alpha(t)=\left(t+t_{c}\right)\left(t^{2}+t_{c}^{2}\right) \sigma_{*},
$$

where $\sigma_{*}=\sigma \varepsilon$-reduced rate of radiation, $\sigma-$
Stefan-Boltzmann constance, $\varepsilon$-coefficient of blackness. The coefficient $\alpha$ is the total coefficient of convective heat transfer $\alpha_{k}$ and coefficient of radiation $\alpha(t)$ in the case of convective heat transfer beam

$$
\alpha=\alpha_{k}+\alpha(t) \text {. }
$$

Transient heat transfer body will be called convective heat transfer with temperature depending on the heat transfer coefficient. The mechanism of that heat transfer is described by the Relation (1).

In the study of processes of heat conduction in solids, which are shaped circular cylinder radius $r_{0}$ using a cylindrical coordinate system $(r, \varphi, z)$. If the temperature is independent of $\varphi$ and $z$ (the case of axial symmetry), the heat equation has the form:

$$
\frac{1}{r} \frac{\partial}{\partial r}\left(r \lambda_{t} \frac{\partial t}{\partial r}\right)=c_{v}(t) \frac{\partial t}{\partial \tau}, 0<r<r_{0}
$$

where $c_{v}(t)$-volumetric heat capacity.

The solution of Equation (4) satisfies the boundary condition of the third kind, the described types of heat transfer are as follows: 


$$
\lambda_{t}(t) \frac{\partial t\left(r_{0}, \tau\right)}{\partial r}+\alpha(t)\left[t\left(r_{0}, \tau\right)-t_{c}\right]=0,
$$

and at $r=0$ satisfies the condition of boundedness of solution, which is equivalent to the condition

$$
\lim _{r \rightarrow 0} \lambda_{t}(t) \frac{\partial t}{\partial r}=0 \text {. }
$$

Besides, the solution of Equation (4) satisfies the initial condition

$$
t(r, 0)=t_{p},
$$

where $t_{p}$ - the initial temperature of the cylinder.

Thus, the mathematical model to determine the temperature field in a circular cylinder has the form of nonlinear boundary value Problems (4)-(7).

For the convenience of calculations in constructing the solution to the Problems (4)-(7) we pass to dimensionless variables. To do this, take the temperature of the heating medium $t_{c}$ by counting the temperature $t_{0}$, and radius $r_{0}$ for the typical size. We introduce the dimensionless temperature $T=t / t_{0}$ and coordinate $\rho=r / r_{0}$. The growth temperature $T-T_{p}$ denoted by $\bar{T}$, where $T_{p}=t_{p} / t_{0}$.

\section{Preliminaries}

Specifications of the material $\lambda_{t}(t), c_{v}(t)$ and heat transfer coefficient $\alpha(t)$ are represented as:

$$
\begin{aligned}
& \lambda_{t}(t)=\lambda_{t 0} \lambda_{t}^{*}(\bar{T}), \\
& c_{v}(t)=c_{v 0} c_{v}^{*}(\bar{T}), \\
& \alpha(t)=\alpha_{0} \alpha^{*}(\bar{T}),
\end{aligned}
$$

where $\lambda_{t 0}, c_{v 0}, \alpha_{0}$-basic coefficients that are relevant dimension, and, $\lambda_{t}^{*}(\bar{T}), c_{v}^{*}(\bar{T}), \alpha^{*}(\bar{T})$-functions that describe the dependence of these characteristics on dimensionless temperature $T$, and

$$
\lambda_{t}^{*}\left(T_{p}\right)=c_{v}^{*}\left(T_{p}\right)=1 .
$$

We introduce the dimensionless time $F O=a_{0} \tau / r_{0}^{2}\left(a_{0}=\lambda_{t 0} / c_{v 0}\right)$ and Bios criterion' $B i=\alpha_{0} r_{0} / \lambda_{t 0}$. That Problems (4)-(7) to increase the temperature in the dimensionless quantity becomes a

$$
\begin{gathered}
\frac{1}{\rho} \frac{\partial}{\partial \rho}\left(\rho \lambda_{t}^{*}(\bar{T}) \frac{\partial \bar{T}}{\partial \rho}\right)=c_{v}^{*}(\bar{T}) \frac{\partial \bar{T}}{\partial F O}, 0<\rho<1, \\
\lambda_{t}^{*}(\bar{T}) \frac{\partial \bar{T}(1, F o)}{\partial \rho}+\operatorname{Bi\alpha }^{*}(\bar{T})\left[\bar{T}(1, F O)-\bar{T}_{c}\right]=0 \\
\lim _{\rho \rightarrow 0} \lambda_{t}^{*}(\bar{T}) \frac{\partial \bar{T}}{\partial \rho}=0, \\
\left.\bar{T}\right|_{F o=0}=0,
\end{gathered}
$$

where $\bar{T}_{c}=T_{c}-T_{p}$.
Nonlinear boundary value Problems (9)-(12) will be solved numerically using the method of lines. We introduce a uniform grid

$$
\omega_{h}=\left\{\rho_{i}=i h ; i=\overline{0, N}, h N=1\right\}
$$

in the interval $0 \leq \rho \leq 1$. The differential operator

$$
\frac{1}{\rho} \frac{\partial}{\partial \rho}\left(\rho \lambda_{t}^{*}(\bar{T}) \frac{\partial \bar{T}}{\partial \rho}\right)
$$

has been replaced by the difference operator [1] at the points of the grid $\rho_{i}, i=\overline{1, N-1}$

$$
\frac{1}{\rho_{i}}\left(\rho_{i-1 / 2} \lambda_{i} \bar{T}_{\bar{\rho}, i}\right)_{\rho, i},
$$

where $\rho_{i-1 / 2}=\rho_{i}-h / 2, \bar{T}_{\bar{\rho}, i}=\left(\bar{T}_{i}-\bar{T}_{i-1}\right) / h$, $\bar{T}_{\rho, i}=\left(\bar{T}_{i+1}-\bar{T}_{i}\right) / h$, and the value $\lambda_{i}$ determined by the formula

$$
\lambda_{i}=\lambda_{t}^{*}\left(\frac{\bar{T}_{i-1}+\bar{T}_{i}}{2}\right)
$$

or

$$
\lambda_{i}=\frac{\lambda_{t}^{*}\left(\bar{T}_{i-1}\right)+\lambda_{t}^{*}\left(\bar{T}_{i}\right)}{2} .
$$

The difference operator (14) approximates the differential operator (13) to the second order in $h$.

We will approach these functions $\bar{T}_{i}=\bar{T}\left(\rho_{i}, F o\right)$ by using the functions $y_{i}=y_{i}(F O), i=\overline{0, N}$, which are based on Equation (9) satisfying the system of ordinary differential equations

$$
\begin{aligned}
& \frac{\mathrm{d} y_{i}}{\mathrm{~d} F O} \\
& =\frac{1}{h \rho_{i} c_{i}}\left[\rho_{i+1 / 2} \lambda_{i+1} \frac{y_{i+1}-y_{i}}{h}-\rho_{i-1 / 2} \lambda_{i} \frac{y_{i}-y_{i-1}}{h}\right], \\
& (i=\overline{1, N-1})
\end{aligned}
$$

where $c_{i}=c_{v}^{*}\left(\bar{T}_{i}\right)$.

We construct the difference boundary condition for the second-order approximation for $\rho=\rho_{N}=1$. The expansion of these values in series Taylor in neighborhood of the point $\rho=\rho_{N}$ is written as follows:

$$
\begin{aligned}
& \frac{\bar{T}_{N}-\bar{T}_{N-1}}{h} \\
& =\bar{T}_{\bar{\rho}, N}=\left.\frac{\partial \bar{T}}{\partial \rho}\right|_{\rho=\rho_{N}}-\left.\frac{h}{2} \frac{\partial^{2} \bar{T}}{\partial \rho^{2}}\right|_{\rho=\rho_{N}}+O\left(h^{2}\right) \rho_{N-1 / 2} \lambda_{N} \\
& =\rho_{N} \lambda_{t}^{*}\left(\bar{T}_{N}\right)-\left.\frac{h}{2} \frac{\partial}{\partial \rho}\left(\rho \lambda_{t}^{*}(\bar{T})\right)\right|_{\rho=\rho_{N}}+O\left(h^{2}\right)
\end{aligned}
$$

Then considering the differential Equation (9) at the point $\rho=\rho_{N}$ we receive 


$$
\begin{aligned}
& \rho_{N-1 / 2} \lambda_{N} \bar{T}_{\bar{\rho}, N} \\
& =\left.\rho \lambda_{t}^{*}(T) \frac{\partial \bar{T}}{\partial \rho}\right|_{\rho=\rho_{N}}-\frac{h}{2}\left[\left.\left.\frac{\partial}{\partial \rho}\left(\rho \lambda_{t}^{*}(\bar{T})\right)\right|_{\rho=\rho_{N}} \frac{\partial \bar{T}}{\partial \rho}\right|_{\rho=\rho_{N}}\right. \\
& \left.+\left.\rho \lambda_{t}^{*}(\bar{T}) \frac{\partial^{2} \bar{T}}{\partial \rho^{2}}\right|_{\rho=\rho_{N}}\right]+O\left(h^{2}\right) \\
& =\left.\rho \lambda_{t}^{*}(\bar{T}) \frac{\partial \bar{T}}{\partial \rho}\right|_{\rho=\rho_{N}}-\frac{h}{2} \rho_{N}\left[\left.\frac{1}{\rho} \frac{\partial}{\partial \rho}\left(\rho \lambda_{t}^{*}(\bar{T}) \frac{\partial \bar{T}}{\partial \rho}\right)\right|_{\rho=\rho_{N}}\right] \\
& +O\left(h^{2}\right) \\
& =\left.\rho \lambda_{t}^{*}(\bar{T}) \frac{\partial \bar{T}}{\partial \rho}\right|_{\rho=\rho_{N}}-\frac{h}{2} \rho_{N}\left[c_{t}^{*}(\bar{T}) \frac{\partial \bar{T}}{\partial F o}\right]_{\rho=\rho_{N}}+O\left(h^{2}\right) .
\end{aligned}
$$

Hence, taking into account that $\bar{T}_{N} \approx y_{N}$, we obtain

$$
\frac{\mathrm{d} y_{N}}{\mathrm{~d} F O}=\frac{2}{h \rho_{N} c_{N}}\left(\rho_{N} \varphi_{N}-\rho_{N-1 / 2} \lambda_{N} y_{\bar{\rho}, N}\right),
$$

where $\varphi_{N}=-\left.B i \alpha^{*}(\bar{T})\right|_{\rho=1}\left(\left.\bar{T}\right|_{\rho=1}-\overline{T_{c}}\right)$.

The difference analog of condition is constructed (11). The values $\bar{T}_{\rho, 0}$ i $\lambda_{1}$ decompose in the Taylor series:

$$
\begin{aligned}
& \bar{T}_{\rho, 0}=\left.\frac{\partial \bar{T}}{\partial \rho}\right|_{\rho=0}+\left.\frac{h}{2} \frac{\partial^{2} \bar{T}}{\partial \rho^{2}}\right|_{\rho=0}+O\left(h^{2}\right), \\
& \lambda_{1}=\lambda_{t}^{*}\left(\bar{T}_{0}\right)+\left.\frac{h}{2} \frac{\partial}{\partial \rho}\left(\lambda_{t}^{*}(\bar{T})\right)\right|_{\rho=0}+O\left(h^{2}\right) .
\end{aligned}
$$

Then

$$
\begin{aligned}
& \lambda_{1} \bar{T}_{\rho, 0} \\
& =\left.\lambda_{t}^{*}(\bar{T}) \frac{\partial \bar{T}}{\partial \rho}\right|_{\rho=0}+\frac{h}{2}\left[\left.\left.\frac{\partial}{\partial \rho}\left(\lambda_{t}^{*}(\bar{T})\right)\right|_{\rho=0} \frac{\partial \bar{T}}{\partial \rho}\right|_{\rho=0}\right. \\
& \left.+\left.\lambda_{t}^{*}(\bar{T}) \frac{\partial^{2} \bar{T}}{\partial \rho^{2}}\right|_{\rho=0}\right]+O\left(h^{2}\right) \\
& =\left.\lambda_{t}^{*}(\bar{T}) \frac{\partial \bar{T}}{\partial \rho}\right|_{\rho=0}+\frac{h}{2}\left[\frac{\partial}{\partial \rho}\left(\lambda_{t}^{*}(\bar{T})\right) \frac{\partial \bar{T}}{\partial \rho}\right]_{\rho=0}+O\left(h^{2}\right)
\end{aligned}
$$

In view of Equation (9) we write

$$
\frac{\partial}{\partial \rho}\left(\lambda_{t}^{*}(\bar{T}) \frac{\partial \bar{T}}{\partial \rho}\right)=-\frac{\lambda_{t}^{*}(\bar{T})}{\rho} \frac{\partial \bar{T}}{\partial \rho}+c_{v}^{*}(\bar{T}) \frac{\partial \bar{T}}{\partial F o} .
$$

Since $\lim _{\rho \rightarrow 0} \frac{\partial \bar{T}}{\partial \rho}=0$, then we have uncertainty

$\left.\frac{\lambda_{t}^{*}(T)}{\rho} \frac{\partial \bar{T}}{\partial \rho}\right|_{\rho=0}$ of type $\frac{0}{0}$. We now turn to the border and reveal uncertainty using the rule of de L'Hospitala, we get

$$
\lim _{\rho \rightarrow 0} \frac{\lambda_{t}^{*}(\bar{T})}{\rho} \frac{\partial \bar{T}}{\partial \rho}=\left[\frac{\partial}{\partial \rho}\left(\lambda_{t}^{*}(\bar{T}) \frac{\partial \bar{T}}{\partial \rho}\right)\right]_{\rho=0} .
$$

Thus from (20) we obtain

$$
\left[\frac{\partial}{\partial \rho}\left(\lambda_{t}^{*}(\bar{T}) \frac{\partial \bar{T}}{\partial \rho}\right)\right]_{\rho=0}=\left.\left.\frac{1}{2} c_{v}^{*}(\bar{T})\right|_{\rho=0} \frac{\partial \bar{T}}{\partial F o}\right|_{\rho=0} .
$$

Taking into account (21), we write (19) as

$$
\lambda_{1} \bar{T}_{\rho, 0}=\left.\left.\frac{h}{4} c_{v}^{*}(\bar{T})\right|_{\rho=0} \frac{\partial \bar{T}}{\partial F o}\right|_{\rho=0}+O\left(h^{2}\right) .
$$

Since the value of $\bar{T}_{0}$ approaches $y_{0}$, then from (22) we obtain the following differential equation at the point $\rho=0$

$$
\frac{\mathrm{d} y_{0}}{\mathrm{~d} F O}=\frac{4}{h c} \lambda_{1} y_{\rho, 0},
$$

where $c_{0}=\left.c_{v}^{*}(\bar{T})\right|_{\rho=0}$.

Thus, we obtain the Cauchy problem for systems of ordinary differential equations approximating the partial derivatives of the space variables with the second-order boundary-value Problems (8)-(11)

$$
\begin{gathered}
\frac{\mathrm{d} y_{0}}{\mathrm{~d} F o}=\frac{4}{c_{0} h^{2}} \lambda_{1}\left(y_{1}-y_{0}\right), \\
\frac{\mathrm{d} y_{i}}{\mathrm{~d} F o}=\frac{1}{h^{2} \rho_{i} c_{i}}\left[\rho_{i+1 / 2} \lambda_{i+1}\left(y_{i+1}-y_{i}\right)-\rho_{i-1 / 2} \lambda_{i}\left(y_{i}-y_{i-1}\right)\right] \\
(i=\overline{1, N-1)}), \\
\frac{\mathrm{d} y_{N}}{\mathrm{~d} F O}=\frac{2}{h \rho_{N} c_{N}}\left(\rho_{N} \varphi_{N}-\rho_{N-1 / 2} \lambda_{N} \frac{y_{N}-y_{N-1}}{h}\right] \\
y_{i}(0)=0
\end{gathered}
$$

Thus, the dependence forms of the heat transfer coefficient $\alpha(t)$, thermal conductivity $\lambda_{t}(t)$ and volumetric heat capacity $c_{v}(t)$ of the selected material are dependent on temperature, then we solve the system numerically (24)-(27). As a result, we obtain the value of temperature increase in grid points $\rho_{i}, i=\overline{1, N}$ along the radius of the cylinder for a given time $F o$.

\section{Thermoelastic State of a Cylinder}

The thermoelastic cylinder state with the activity of the found axisymmetric temperature field are defined by three non-zero components of stress tensor $\sigma_{r r}, \sigma_{\varphi \varphi}, \sigma_{z z}$, which in dimensionless form is rewritten as:

$$
\left\{\sigma_{\rho}, \sigma_{\phi}, \sigma_{\zeta}\right\}=\frac{\left\{\sigma_{r r}, \sigma_{\varphi \varphi}, \sigma_{z z}\right\}}{2 G_{0} \alpha_{t 0} t_{o}},
$$

where $G_{0}$ i $\alpha_{t 0}$-supporting values of the shear mo- 
dulus and coefficient of linear thermal expansion.

However, they are expressed through the dimensionless radial displacement $\bar{u}=\frac{u}{r_{0} \alpha_{t 0} t_{o}}$, so

$$
\begin{array}{r}
\sigma_{\rho}=\bar{G}(T)\left[(1-v(\bar{T})) \frac{\partial \bar{u}}{\partial \rho}+v(\bar{T})\left(\frac{\bar{u}}{\rho}+e_{\zeta}\right)\right. \\
\left.-(1-v(\bar{T})) \Phi^{*}(\bar{T})\right] \\
\sigma_{\phi}=\bar{G}(T)\left[v(\bar{T}) \frac{\partial \bar{u}}{\partial \rho}+(1-v(\bar{T})) \frac{\bar{u}}{\rho}+v(\bar{T}) e_{\zeta}\right. \\
\left.-(1-v(\bar{T})) \Phi^{*}(\bar{T})\right],
\end{array}
$$

$$
\begin{aligned}
& \sigma_{\zeta} \\
& =\bar{G}(T)\left[v(\bar{T})\left(\frac{\partial \bar{u}}{\partial \rho}+\frac{\bar{u}}{\rho}\right)+(1-v(\bar{T}))\left(e_{\zeta}-\Phi^{*}(\bar{T})\right)\right]
\end{aligned}
$$

and satisfy the equation of balance

$$
\frac{\partial \sigma_{\rho}}{\partial \rho}+\frac{\sigma_{\rho}-\sigma_{\phi}}{\rho}=0 .
$$

Here $e_{\zeta}=\frac{e_{z z}}{\alpha_{t 0} t_{o}}$, where $e_{z z}$-was the axial strain; $\bar{G}(T)=\frac{G^{*}(\bar{T})}{1-2 v(\bar{T})}, \Phi^{*}(\bar{T})=\frac{1+v(\bar{T})}{1-v(\bar{T})} \int_{0}^{\bar{T}} \alpha_{t}^{*}(\xi) \mathrm{d} \xi$,

$\left(v(\bar{T}), \alpha_{t}^{*}(\bar{T}), G^{*}(\bar{T})\right.$-the function describing the dependence of the Poisson coefficient of the linear thermal expansion and modulus of the dimensionless temperature increase. If we substitute the dependence (28)-(30) into equilibrium Equation (31), we get the differential equation

$$
\begin{aligned}
& \frac{\partial}{\partial \rho}\left(\frac{1}{\rho} \frac{\partial}{\partial \rho}(\rho \bar{u})\right) \\
& =\frac{\partial \Phi^{*}(\bar{T})}{\partial \rho}-n(T) e_{\zeta}-\psi(T)\left(\frac{\partial \bar{u}}{\partial \rho}+m(T) \frac{\bar{u}}{\rho}-\Phi^{*}(\bar{T})\right),
\end{aligned}
$$

where

$$
\begin{aligned}
& \psi(T)=\frac{\partial}{\partial \rho}(\ln (\bar{G}(T)(1-v(T)))), \\
& m(T)=\frac{\partial}{\partial \rho}(\bar{G}(T) v(\bar{T})) / \frac{\partial}{\partial \rho}(\bar{G}(T)(1-v(\bar{T}))), \\
& n(T)=\frac{1}{\bar{G}(T)(1-v(\bar{T}))} \frac{\partial(\bar{G}(T) v(\bar{T}))}{\partial \rho} .
\end{aligned}
$$

The solution of Equation (32), which satisfies the condition of the limited movement of the cylinder axis, found by perturbation [1-3] has the form of:

$$
\bar{u}=\sum_{k=0}^{\infty} \bar{u}_{k},
$$

where

$$
\begin{aligned}
\bar{u}_{0}=c_{10} \rho & +\frac{1}{\rho} H^{*}(\rho)+\frac{1}{2}\left(\rho H_{\psi}^{(0)}(\rho)-\frac{1}{\rho} H_{\psi}^{(2)}(\rho)\right) \\
- & \frac{e_{\zeta 0}}{2}\left(\rho H_{n}^{(0)}(\rho)-\frac{1}{\rho} H_{n}^{(2)}(\rho)\right) \\
\bar{u}_{k}= & c_{1 k} \rho-\frac{e_{\zeta k}}{2}\left(\rho H_{n}^{(0)}(\rho) \frac{1}{\rho} H_{n}^{(2)}(\rho)\right) \\
& -\frac{1}{2}\left(\rho H_{k-1}^{(0)}(\rho) \frac{1}{\rho} H_{k-1}^{(2)}(\rho)\right),(k \geq 1) \\
& H^{*}(\rho)=\int_{0}^{\rho} \rho \Phi^{*}(\bar{T}) \mathrm{d} \rho \\
& H_{\psi}^{(m)}(\rho)=\int_{0}^{\rho} \rho^{m} \psi(\rho) \bar{\Phi}^{*}(T) \mathrm{d} \rho \\
& H_{n}^{(m)}(\rho)=\int_{0}^{\rho} \rho^{m} n(\rho) \mathrm{d} \rho \\
& H_{k-1}^{(m)}(\rho)=\int_{0}^{\rho} \rho^{m} f_{k-1}(\rho) \mathrm{d} \rho \\
f_{k-1}(\rho)= & \psi(T)\left(\frac{\partial \bar{u}_{k-1}}{\partial \rho}+m(T) \frac{\bar{u}_{k-1}}{\rho}\right) .
\end{aligned}
$$

According to (33) the thermal stress $\sigma_{\rho}, \sigma_{\phi}, \sigma_{\zeta}$, is found by the formulas:

$$
\left\{\sigma_{p}, \sigma_{\phi}, \sigma_{\zeta}\right\}=\sum_{k=0}^{\infty}\left\{\sigma_{p k}, \sigma_{\phi k}, \sigma_{\zeta k}\right\},
$$

where

$$
\begin{aligned}
\sigma_{\rho 0}=\bar{G}(T)\left[c_{10}-\frac{(1-2 v(\bar{T})) H^{*}(\rho)}{\rho^{2}}\right. \\
\left.+H_{\psi}^{+}(\rho)+e_{\zeta 0}\left(v(\bar{T})-H_{n}^{+}(\rho)\right)\right],
\end{aligned}
$$

$$
\begin{aligned}
& \sigma_{p k} \\
& =\bar{G}(T)\left[c_{1 k}+e_{\zeta k}\left(v(\bar{T})-H_{n}^{+}(\rho)\right)-H_{k-1}^{+}(\rho)\right],(k \geq 1),
\end{aligned}
$$

$$
\begin{gathered}
\sigma_{\phi 0} \\
=\bar{G}(T)\left[c_{01}-\frac{(1-2 v(\bar{T})) H^{*}(\rho)}{\rho^{2}}-(1-2 v(\bar{T})) \Phi^{*}(\bar{T})\right. \\
\left.+H_{\psi}^{-}(\rho)+e_{\zeta 0}\left(v(\bar{T})-H_{n}^{-}(\rho)\right)\right],
\end{gathered}
$$




$$
\begin{aligned}
& \sigma_{\phi k} \\
& =\bar{G}(T)\left[c_{1 k}+e_{\zeta k}\left(v(\bar{T})-H_{n}^{-}(\rho)\right)-H_{k-1}^{-}(\rho)\right],(k \geq 1)
\end{aligned}
$$

$$
\begin{aligned}
& \sigma_{\zeta 0} \\
& \begin{aligned}
=\bar{G}(T)[ & 2 v(\bar{T}) c_{01}-(1-2 v(\bar{T})) \Phi^{*}(\bar{T})+v(\bar{T}) H_{\Psi}^{(0)}(\rho) \\
& \left.+e_{\zeta 0}\left(1-v(\bar{T})-v(\bar{T}) H_{n}^{(0)}(\rho)\right)\right],
\end{aligned}
\end{aligned}
$$

$$
\begin{array}{r}
\sigma_{\zeta k} \\
=\bar{G}(T)\left[2 v(\bar{T}) c_{1 k}+e_{\zeta k}\left(1-v(\bar{T})-v(\bar{T}) H_{n}^{(0)}(\rho)\right)\right. \\
\left.-v(\bar{T}) H_{k-1}^{(0)}(\rho)\right],(k \geq 1)
\end{array}
$$

where

$$
\begin{array}{r}
H_{\eta}^{ \pm}(\rho)=\frac{1}{2}\left(H_{\eta}^{(0)}(\rho) \pm \frac{1-2 v(\bar{T})}{\rho^{2}} H_{\eta}^{(2)}(\rho)\right), \\
(\eta=\psi, n, k-1) .
\end{array}
$$

The sustainable integration $c_{1 k}(k \geq 0)$ and parts of the development of axial strain $e_{\zeta k}(k \geq 0)$ determine the conditions on the outer surface $\left.\sigma_{\rho k}\right|_{\rho=1}=0$ and relations for unmounted ends of the cylinder $\int_{0}^{1} \rho \sigma_{\zeta k} \mathrm{~d} \rho=0$, by solving system of linear equations

$$
A X_{k}=B_{k},(k \geq 0),
$$

where

$$
\begin{aligned}
& \boldsymbol{A}=\left(\begin{array}{ll}
a_{11} & a_{12} \\
a_{21} & a_{22}
\end{array}\right), \boldsymbol{X}_{k}=\left(\begin{array}{c}
c_{1 k} \\
e_{\zeta k}
\end{array}\right), \boldsymbol{B}_{k}=\left(\begin{array}{l}
b_{1 k} \\
b_{2 k}
\end{array}\right) \\
& a_{11}=1, a_{12}=v_{1}-H_{n}^{+}\left(\rho_{1}\right) \\
& a_{21}=2 \int_{0}^{\rho_{1}} \rho \bar{G}(T) v(\bar{T}) \mathrm{d} \rho \\
& a_{22}=\int_{0}^{\rho_{1}} \rho \bar{G}(T)\left(1-v(\bar{T})-v(\bar{T}) H_{n}^{(0)}(\rho)\right) \mathrm{d} \rho \\
& b_{10}=\bar{v}_{1} H^{*}\left(\rho_{1}\right)-H_{\psi}^{+}\left(\rho_{1}\right) \\
& b_{20}=\int_{0}^{\rho_{1}} \rho \bar{G}(T)\left(\overline{v_{1}} \Phi^{*}(T)-v(\bar{T}) H_{\psi}^{(0)}(\rho)\right) \mathrm{d} \rho \\
& b_{1 k}=H_{k-1}^{+}\left(\rho_{1}\right), \\
& b_{2 k}=\int_{0}^{\rho_{1}} \rho \bar{G}(T) v(\bar{T}) H_{k-1}^{(0)}(\rho) \mathrm{d} \rho .
\end{aligned}
$$

Here

$$
\rho_{1}=1, v_{1}=\left.v(\bar{T})\right|_{\rho=1}, \overline{v_{1}}=1-2 v_{1}, G_{1}=\left.\bar{G}(T)\right|_{\rho=1} .
$$

For fixed ends of the cylinder we have $e_{\zeta}=0$.

If the thermomechanical properties of the material the cylinder does not depend on temperature, then transfer and thermal stresses are calculated by the formulas:

$$
\bar{u}_{i}=\frac{1+v_{i}}{1-v_{i}} \frac{\alpha_{t i}^{*}}{\rho} \int_{0}^{\rho} \rho \bar{T}_{i}(\rho, F o) \mathrm{d} \rho+c_{i} \rho,
$$

$$
\begin{aligned}
& \sigma_{\rho i} \\
& =G_{i}^{*}\left[-\frac{1+v_{i}}{1-v_{i}} \frac{\alpha_{t i}^{*}}{\rho^{2}} \int_{0}^{\rho} \rho \bar{T}_{i}(\rho, F o) \mathrm{d} \rho+\frac{1}{1-2 v_{i}}\left(\tilde{n}_{i}+v_{i} e_{\zeta i}\right)\right],
\end{aligned}
$$

$\sigma_{\phi i}$

$$
\begin{aligned}
& =G_{i}^{*}\left[\frac{\left(1+v_{i}\right) \alpha_{t i}^{*}}{1-v_{i}}\left(\frac{1}{\rho^{2}} \int_{0}^{\rho} \rho \bar{T}_{i}(\rho, F o) \mathrm{d} \rho+\bar{T}_{i}(\rho, F o)\right)\right. \\
& \left.\quad+\frac{1}{1-2 v_{i}}\left(\tilde{n}_{i}+v_{i} e_{\zeta i}\right)\right], \\
& \sigma_{\zeta i}= \\
& \frac{G_{i}^{*}}{1-2 v_{i}}\left[2 v_{i} c_{i}+\left(1-v_{i}\right) e_{\zeta i}+\frac{1+v_{i}}{1-v_{i}}\left(2 v_{i}-1\right) \alpha_{t i}^{*} \bar{T}_{i}(\rho, F o)\right],
\end{aligned}
$$

where $c_{i}=\frac{1-3 v_{i}}{1-v_{i}} \frac{\alpha_{t i}^{*}}{\rho_{1}^{2}} \int_{0}^{\rho_{1}} \rho \bar{T}_{i}(\rho, F o) \mathrm{d} \rho$,

$$
e_{\zeta i}=\frac{1\left(1+v_{i}\right)\left(1-2 v_{i}\right)}{\left(1-v_{0}-2 v_{i}^{2}\right)} \frac{\alpha_{t i}^{*}}{\rho_{1}^{2}} \int_{0}^{\rho_{1}} \rho \bar{T}_{i}(\rho, F o) \mathrm{d} \rho,
$$

$\bar{T}_{i}(\rho, F o)$-increase of temperature in the cylinder for constant thermophysical characteristics; $\alpha^{*}, G_{i}^{*}$-steel coefficient of linear thermal expansion and modulus taken at the initial or maximum temperature, or mean integral value of the selected temperature range.

\section{Numerical Study}

This section investigated the temperature field and thermoelastic state of thermosensitive infinite circular cylinder. Radiant heat exchange with the environment at a temperature $t_{c}$ is due to the surface $r=r_{0}$ of the cylinder, which is free from power loads. In that case, the radiant heat exchange has been formed to convective heat transfer coefficient in the form of (2). The initial temperature of the cylinder is $t_{p}=300 \mathrm{~K}$. The medium temperature $t_{c}$ is equal to $1100 \mathrm{~K}$ and it is selected by supporting. Titanium alloy Ti-6Al-4V taken by the material. Temperature dependence of thermal and mechanical properties of the alloy are the form [4] in the temperature range from $300 \mathrm{~K}$ to $1100 \mathrm{~K}$. 


$$
\begin{gathered}
\lambda_{t}(t)=1.1+0.017 t[\mathrm{~W} /(\mathrm{m} \cdot \mathrm{K})] \\
c_{v}(t)=c_{p}(t) \rho(t), \\
c_{p}(t)=3.5 \times 10^{2}+8.78 \times 10^{-1} t \\
-9.74 \times 10^{-4} t^{2}+4.43 \times 10^{-7} t^{3}[\mathrm{~kJ} / \mathrm{kg} \cdot \mathrm{K}], \\
\rho=4420.0 /\left\{1.0+\alpha_{t}(t)(t-300.0)\right\}\left[\mathrm{kg} / \mathrm{m}^{3}\right] \\
\alpha_{t}(t) \quad \\
=7.43 \times 10^{-6}+5.56 \times 10^{-9} t-2.69 \times 10^{-12} t^{2}[1 / \mathrm{K}], \\
E(t)=122.7-0.0565 t[\mathrm{GPa}] \\
v(t)=0.2888+32.0 \times 10^{-6} t .
\end{gathered}
$$

For the purpose of comparison, we studied the temperature field and caused it thermoelastic stable in the same noetherian sensitive cylinder under constant thermal and mechanical characteristics of titanium alloy.

This steel coefficient of thermal conductivity $\lambda_{t}(t)$, volumetric heat capacity $c_{v}(t)$, modulus $G(t)$, coefficient of linear thermal expansion $\alpha_{t}(t)$ and coefficient of Poisson $v(t)$, we take as: $a$ ) the initial temperature $t_{p}=300 \mathrm{~K}$, and $b$ ) the maximum temperature of $t_{c}=$ $1100 \mathrm{~K}, c)$ mean-integral temperature range from 300 $1100 \mathrm{~K}$, under

$$
\chi_{c}=\frac{1}{t_{\tilde{n}}-t_{p}} \int_{t_{p}}^{t_{\tilde{n}}} \chi^{*}(\xi) \mathrm{d} \xi .
$$

The results of numerical studies of the temperature $\bar{T}$ growth, which were obtained in dimensionless form, are presented in graphs in Figures 1-2.

The distribution rates of temperature along the radial coordinate cylinder for values $F O=0.1$ of the Fourier criterion are shown in Figure 1. The dependence of the temperature growth on the criterion of Fourier on the surface $\rho=1$ of the cylinder is illustrated in Figure 2. Here curve 1 correspond to the results of calculations taking into account the temperature dependence of ther-

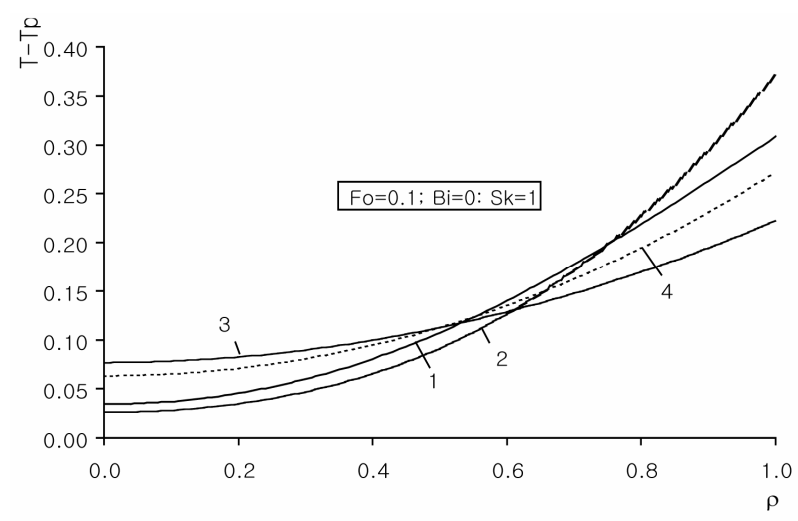

Figure 1. Temperature dependence of the radial coordinate. mophysical characteristics of titanium alloy (48)-(51), curve 2 -at $\lambda_{t}(t)=\lambda_{t}\left(t_{\max }\right), c_{v}(t)=c_{v}\left(t_{\max }\right)$; curve 3 —at $\lambda_{t}(t)=\lambda_{t}\left(t_{\max }\right), \quad c_{v}(t)=c_{v}\left(t_{\max }\right)$; curve 4 -for sustainable medium-integer values of material from a selected range of temperature. The analysis of the research shows that the largest discrepancy between the temperature growth in the cylinder taking into account the temperature dependence of thermophysical characteristics of materials and for stable values of characteristics is in the case $\lambda_{t}(t)=\lambda_{t}\left(t_{\max }\right), c_{v}(t)=c_{v}\left(t_{\max }\right)$.

As it is visible from the graphs in Figure 2 the maximum difference between the increases of temperature $\bar{T}$ on the surface $\rho=1$ of the cylinder by taking into account the temperature dependence of thermophysical of characteristics material (curve 1) and stable mediuminteger values of thermal conductivity and volumetric heat capacity (curve 4) does not exceed 10\% (for Fo $>0.1$ ).

Figures 3-9 show graphs of distributions of displacement and stress tensor component along the radial coordinate $\rho$ for the Fourier criterion $F O=0.1$ and on the surface of the cylinder $\rho=1$ depending on Fo.

Here curve 1 correspond to the results of calculations taking into account the temperature dependence of thermal and mechanical characteristics of titanium alloy (48)-(53).

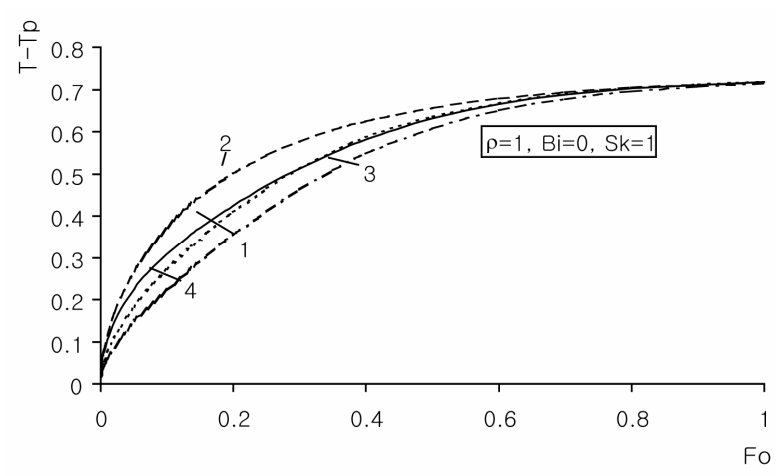

Figure 2. Temperature dependence of the criterion of the Fourier.

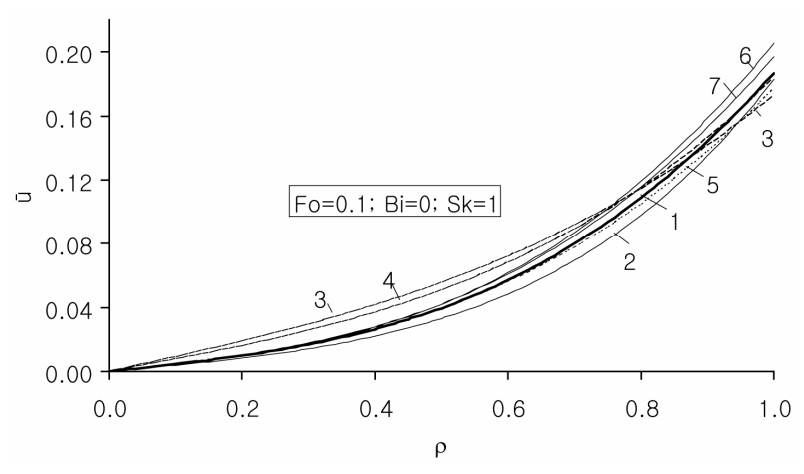

Figure 3. Temperature dependence of the radial coordinate. 


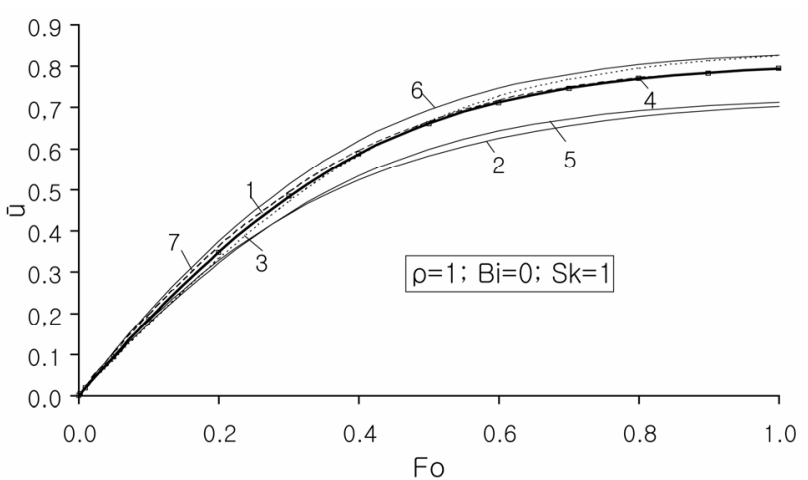

Figure 4. Temperature dependence of the criterion of the Fourier.

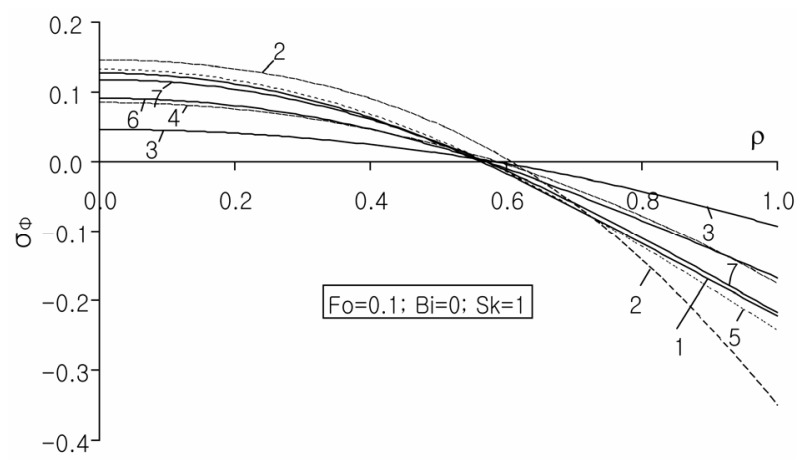

Figure 5. Temperature dependence of the radial coordinate.

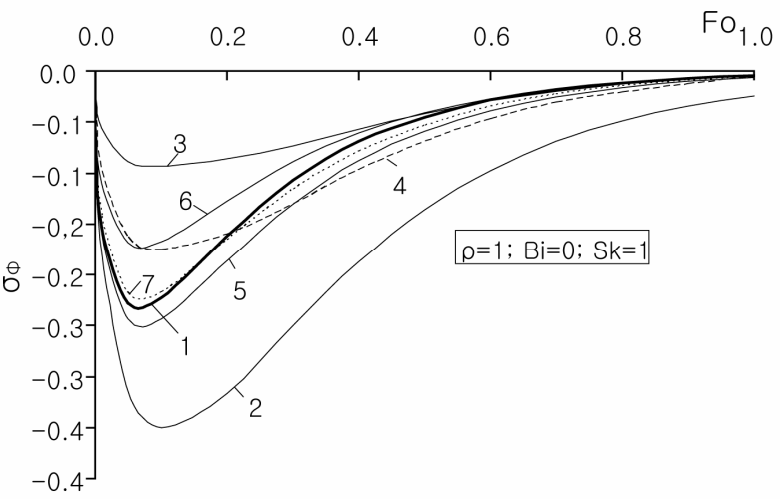

Figure 6. Temperature dependence of the criterion of the Fourier.

The curves 2 - 4 correspond to displacement or stress, found by constant values of thermal and mechanical properties of the material: curve 2-initially under the temperature curve 3-the maximum temperature curve 4-for sustainable medium-integer values of titanium alloy with a temperature range $300-1100 \mathrm{~K}$ in Figures 3-9. In the form of curves 5 - 7 there are the data distributions for the temperature-dependent thermal properties shown $\lambda_{t}(t), c_{v}(t)$, and constant values of mechanical properties (which are taken for the initial (curve 5), and the maximum temperature (curve 6)) and sustainable me-

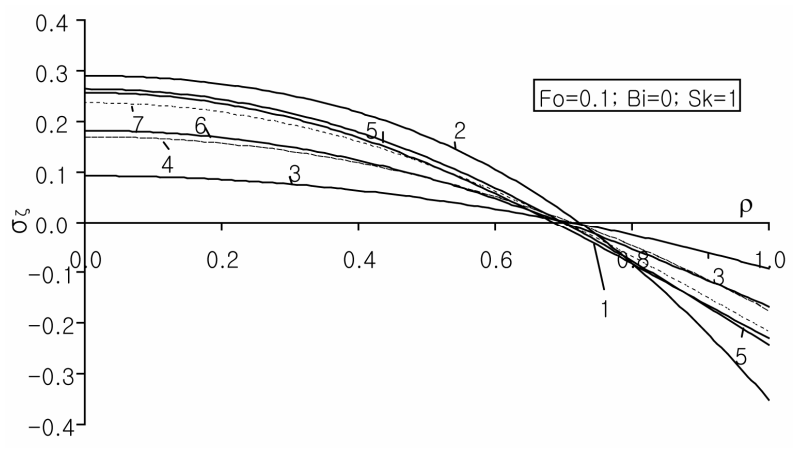

Figure 7. Temperature dependence of the radial coordinate.

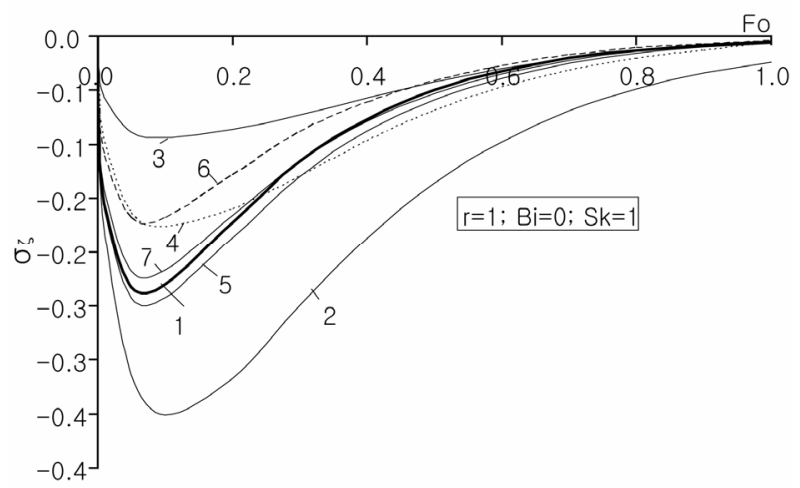

Figure 8. Temperature dependence of the criterion of the Fourier.

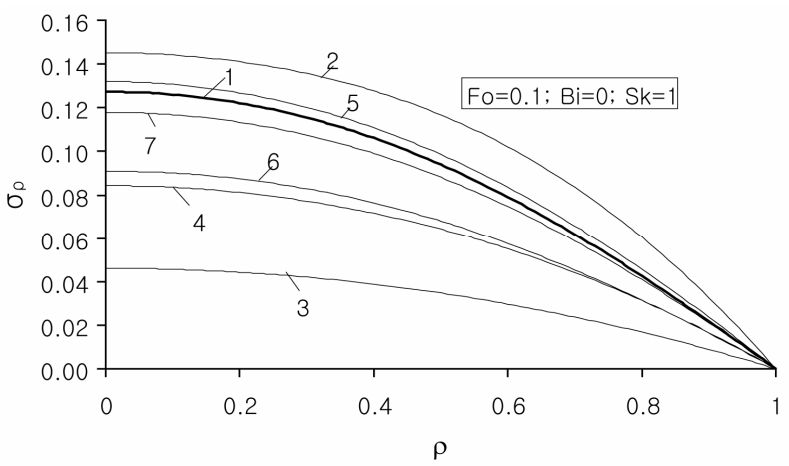

Figure 9. Temperature dependence of the radial coordinate.

dium integer values of mechanical characteristics of titanium alloy with temperature range 300 - $1100 \mathrm{~K}$ (curve 7).

The analysis of differences between the values of both displacements and stresses, which are calculated for all dependent on the temperature characteristics of the material (heat-sensitive body), and their values calculated by the constant characteristics (not heat-sensitive body) indicates that:

- they exceed $60 \%$, if the properties take non heat-sensitive value for thermo-sensitive characteristics at maximum temperature;

- within $12 \%$ - $40 \%$ when take on characteristics non 
heat-sensitive body thermo-sensitive properties at the initial temperature;

- they are within $4 \%-20 \%$, when the characteristics non heat-sensitive body take mid-integral values of thermal and mechanical characteristics. Then the maximum difference between them exceeds $60 \%$.

Thus, studies show that by ignoring the temperature dependence of thermal and mechanical characteristics of the material, the distribution of temperature field and defined by its thermo-elastic state of the body that differ significantly from the true can be achieved.

The likely thermoelastic state of structural elements of modern technology, which in the process of their manufacture and operation exposed to high heat or cooled to low temperatures, preferably determined, based on the model of thermosensitive bodies [5,6]. In that model we consider the temperature dependence of thermal and mechanical properties of the material. That mathematical model to determine the thermoelastic parameters is significantly more complicated in comparison with the same model by neglecting thermo-sensitive material. The temperature is determined from the nonlinear problem, which is not only nonlinear heat equation and boundary conditions and in case of default on the body surface heat flux, convective or convective-radiation heat transfer [7,8]. The corresponding thermoelasticity problem is the same boundary value problem for differential equations with variable coefficients [5]. For these mathematical models the construction of solution is usually carried out by numerical methods [9].

\section{REFERENCES}

[1] J. J. Burak and R. M. Kushnir, "Modeling and Optimization in Termomehanitsi Electrically Inhomogeneous Bod- ies," In: J. J. Burak and R. M. Kushnir, Eds., Thermoelasticity Thermosensitive Bodies, Vol. 3, Lviv, 2009, p. 412.

[2] V. A. Lomakin, "The Theory of Elasticity of Inhomogeneous Bodies," Moscow State University Press, Moscow, 1976, p. 367.

[3] N. H. Moiseev, "The Asymptotic Methods of Nonlinear Mechanics," Nauka, Moscow, 1981, p. 400.

[4] R. M. Kushnir and V. Popovycz, "Stressed State Thermosensitive Body Rotation in the Plane Axialsymmetric Temperature Field," Visnuk Dnipropetrovskoho University, Median Mechanics, No. 2, 2006, pp. 91-96.

[5] Y. Tanigawa, T. Akai, R. Kawamura and N. Oka, "Transient Heat Conduction and Thermal Stress Problems of a Nonhomogeneous Plate with Temperature-Dependent Material Properties," Journal of Thermal Stresses, Vol. 19, No. 1, 1996, pp. 77-102. doi:10.1080/01495739608946161

[6] N. Noda, "Thermal Stresses in Materials with Temperature-Dependent Properties," Thermal Stresses Journal, 1986, pp. 391-483.

[7] V. S. Popovych and H. Yu Harmatiy, "Analytical and Numerical Methods of Solutions of Heat Conduction Problems with Temperature-Sensitive Body Convective Heat Transfer," Problems of Mechanics and Mathematics, Pidstryhach, No. 13-93, Lviv, 1993, p. 67.

[8] H. Yu. Harmatiy, M. B. Kutniv and V. S. Popovich, "Numerical Solution of Unsteady Heat Conduction Problems with Temperature-Sensitive Body Convective Heat Transfer," Engineering, No. 1, 2002, pp. 21-25.

[9] A. Samarskiy, "The Theory of Difference Schemes," Nauka, Moscow, 1977, p. 656. 